\title{
LES APPLICATIONS OPTIQUES DES VERRES SEMI-CONDUCTEURS
}

\section{J. CORNET}

Laboratoires d'Electronique et de Physique Appliquée

3, avenue Descartes, 94450 Limeil-Brévannes, France

\begin{abstract}
Résumé. - Un effort important a été consacré ces dernières années à l'étude des propriétés des verres semi-conducteurs (V. S. C.). Parmi ceux-ci, ce sont surtout les composés à base de soufre, sélénium ou tellure qui ont pris une importance technologique, essentiellement à cause de leurs propriétés optiques ou opto-électroniques. On décrit différentes applications, en particulier celles basées sur la photoconduction et sur les modifications d'ordre local dans le matériau vitreux soumis à une irradiation lumineuse. Enfin les V. S. C. semblent avoir un avenir prometteur en acoustooptique et en optique intégrée.
\end{abstract}

Abstract. - A large effort has been devoted recently to the investigation of properties of semiconducting glasses (S. C. G.). Sulfure, selenium and tellurium-based glasses received most attention due to their potential technological importance in applications dealing with their optical or optoelectrical properties. Some applications are described especially those involving photoconduction or the modification of local order in light-irradiated glasses. Finally, it seems that S. C. G. could have some future in the acousto-optical field or in integrated optics.

1. Introduction. - Depuis une vingtaine d'années, les verres semi-conducteurs ont reçu un intérêt croissant, car ils ont montré des propriétés potentiellement utilisables : certains composés présentent en effet une excellente transmission optique de 3 à $14 \mu$ et de nombreux éléments d'optique infrarouge ont pu être réalisés [1]. En outre, les propriétés de commutation et de mémoire bistable sous l'effet d'un champ électrique ont amené une flambée d'intérêt vers les années 60 , ne serait-ce que parce qu'elles permettaient d'envisager la fabrication de dispositifs de commande électrique insensibles aux radiations et à prix de revient réduit [2].

En fait, les applications purement électroniques n'ont jamais atteint une extension considérable car elles se sont montrées assez peu fiables et elles se sont heurtées à une concurrence sévère de la part de dispositifs à base de matériaux cristallins. Inversement, les applications optiques ou opto-électroniques ont pris une grande importance, soit qu'elles utilisent une propriété spécifique, soit que le processus de fabrication est simple et bon marché. Ce sont ces applications que nous présenterons dans cet article, après avoir résumé les principales propriétés des verres semi-conducteurs.

2. Propriétés des verres semi-conducteurs (V.S. C.). - 2.1 GÉNÉRALITÉs. - Ce sont les éléments silicium, germanium et ceux de la colonne de l'oxygène $(\mathrm{S}, \mathrm{Se}$, Te) purs ou alliés avec les éléments des colonnes III à VI de la classification périodique qui peuvent être retenus à l'état désordonné et présentent dans cet état un caractère semi-conducteur. Pour les obtenir à partir de l'état liquide, il suffit de refroidir le liquide à une vitesse supérieure à la vitesse critique de trempe pour le liquide considéré ; cette vitesse est définie par la tangente à la courbe TTT (température, temps, transformation) correspondant à la nucléation (Fig. 1). Lorsque la tension interfaciale liquide-cristal et/ou la viscosité du liquide sont élevées, le temps d'incubation pour la nucléation au voisinage du nez de la courbe TTT est suffisant pour que le matériau soit retenu à l'état vitreux par refroidissement lent. Dans d'autres cas,

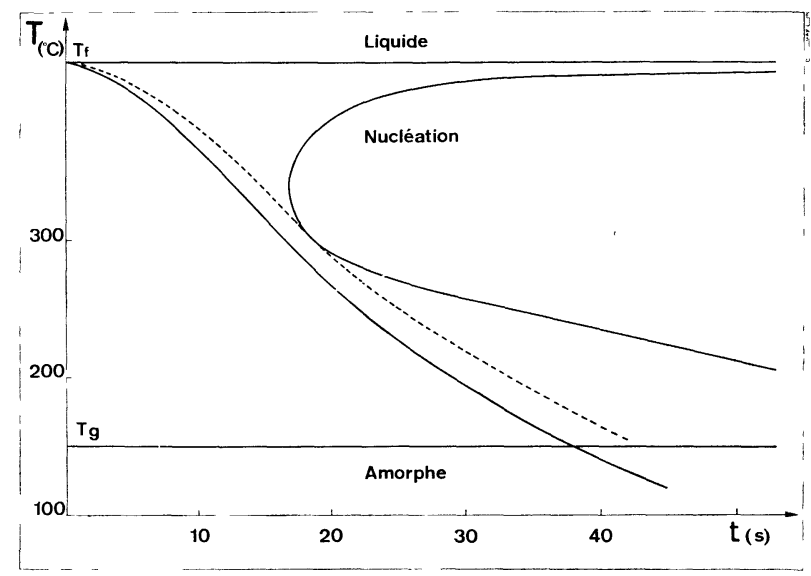

Fig. 1. - Courbe TTT schématique correspondant à la nucléation dans le composé $\mathrm{Ge}_{20} \mathrm{Te}_{80}$. La trempe du liquide suivant la courbe de refroidissement expérimentale (traits pleins) conduit à la vitrification. La courbe en tirets permet de définir la vitesse critique de trempe (d'après [8]). 
particulièrement lorsque les liaisons intra-moléculaires présentent un caractère métallique, il peut être difficile, voire impossible, de préparer le verre par trempe du liquide; il faut alors recourir à l'une des diverses méthodes de déposition en phase vapeur.

Il n'y a généralement pas de différence qualitative ou quantitative entre les propriétés structurales, optiques ou de transport des verres préparés soit sous forme massive, soit en couche mince : on dit que le verre a été préparé dans son état idéal ; les seuls écarts à l'idéalité concernent $\mathrm{Ge}$ et $\mathrm{Si}$ amorphes pour lesquels il existe un certain nombre de liaisons coupées dépendant de la méthode de préparation et des recuits éventuels ultérieurs [3]. Il est donc possible de mesurer une propriété donnée indifféremment sur un échantillon massif ou une couche mince ; cependant, certaines propriétés ne peuvent être mesurées qu'à l'état massif (dureté, diffusion des neutrons...), d'autres à l'état de couche mince (diffusion des électrons...), d'autres enfin nécessitent la préparation aussi bien d'échantillons massifs que de couches minces (absorption optique dans le visible et l'infrarouge).

Les verres semi-conducteurs sont généralement dans un état métastable : ils ont tendance à cristalliser avec une cinétique dépendant de la température, de la viscosité, de la tension interfaciale amorphe-cristal et de l'enthalpie libre de cristallisation (Fig. 2). Les phases

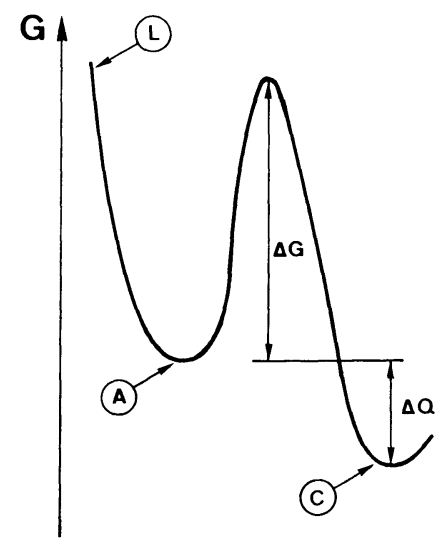

FIG. 2. - Diagramme schématique d'énergie caractérisant un matériau vitreux. $L, A$ et $C$ désignent respectivement l'enthalpie libre $G$ du liquide, du verre et du matériau après cristallisation. $\Delta G$ et $\Delta Q$ désignent l'enthalpie d'activation pour la cristallisation et l'énergie récupérée au cours de la cristallisation.

de cristallisation présentent un gap voisin de celui des composés amorphes s'il n'y a pas de variation sensible d'ordre local entre les deux états. C'est le cas de l'ensemble des V.S. C. (Tableau I), sauf du tellure et de ses composés qui présentent à l'état cristallin un caractère métallique très marqué alors qu'à l'état amorphe les liaisons sont essentiellement covalentes. Il en résulte que ce n'est que pour les verres à base de tellure qu'il y a, au cours de la cristallisation, de grandes variations de nombreuses propriétés physiques en particulier de l'absorption optique [4].

\section{TABleaU I}

Comparaison des gaps optiques $E_{\mathbf{G}}$ (définis par $\alpha=10^{3} \mathrm{~cm}^{-1}$ à la température ordinaire) pour plusieurs éléments et composés à l'état cristallin et à l'état amorphe (d'après [5]). Le contraste optique en transmission $C=\frac{\tau_{\max }-\tau_{\min }}{\tau_{\min }}$ entre les deux états est calculé pour une couche de $1000 \AA$ irradiée avec des photons d'énergie égale au gap optique de la phase la moins opaque (transmittance $\tau_{\max }$ ). $\tau_{\min }$ désigne la transmittance de la phase la plus opaque.

\begin{tabular}{|c|c|c|c|}
\hline \multirow{3}{*}{ Nature } & \multicolumn{2}{|c|}{$E_{\mathrm{G}}(\mathrm{eV})$} & \multirow{3}{*}{ C $(\%)$} \\
\hline & & & \\
\hline & cristallin & amorphe & \\
\hline - & - & - & - \\
\hline $\mathrm{Ge}$ & 0,75 & 0,65 & 10 \\
\hline $\mathrm{Si}$ & 1,5 & $1,3\left({ }^{1}\right)$ & 2 \\
\hline $\mathrm{Se}$ & 1,8 & 1,85 & 2 \\
\hline $\mathrm{Te}$ & 0,4 & 0,8 & 60 \\
\hline $\mathrm{As}_{2} \mathrm{~S}_{3}$ & 2,62 & 2,4 & 10 \\
\hline $\mathrm{As}_{2} \mathrm{Se}_{3}$ & 1,85 & 1,7 & 4 \\
\hline
\end{tabular}

(1) Après recuit à $396^{\circ} \mathrm{C}$ (cf. [5]).

Les V. S. C. présentent cependant, dans tous les cas, deux différences fondamentales avec les composés cristallins correspondant [5] :

- La mobilité de déplacement (Drift Mobility) des porteurs des deux signes est très faible à cause de la très forte densité des pièges superficiels qui provoquent, durant le transit d'un porteur, des allées et venues incessantes entre états délocalisés et états pièges, d'énergie voisine. La mobilité la plus élevée mesurée dans un V. S. C. (trous dans le sélénium pur) est 100 à 1000 fois plus faible que dans un semi-conducteur cristallin de haute pureté.

- Il y a un continuum d'états liés dans le gap. Ces états sont dus au désordre et sont occupés jusqu'au niveau de Fermi situé au milieu du gap. Les électrons localisés sur les états de milieu du gap peuvent sauter d'un site à l'autre par effet tunnel assisté par les phonons (hopping). Mais ce saut nécessite une énergie finie si bien que ce processus de conduction entre états localisés se fait avec une mobilité très réduite et ne peut être observé qu'à basse température. Il est donc justifié d'introduire un pseudo-gap optique $E_{\mathrm{G}}$ distinct d'un pseudo-gap de mobilité $E_{\varphi}$ sensiblement égal à deux fois l'énergie d'activation pour la conductivité électrique à haute température (modèle de Mott [5]).

2.2 ABSORPTION OPTIQUE. - Il résulte des propriétés générales précédentes que la courbe d'absorption optique, au lieu de présenter une discontinuité comme pour les semi-conducteurs intrinsèques à gap direct, présente l'allure générale de la figure 3 : 


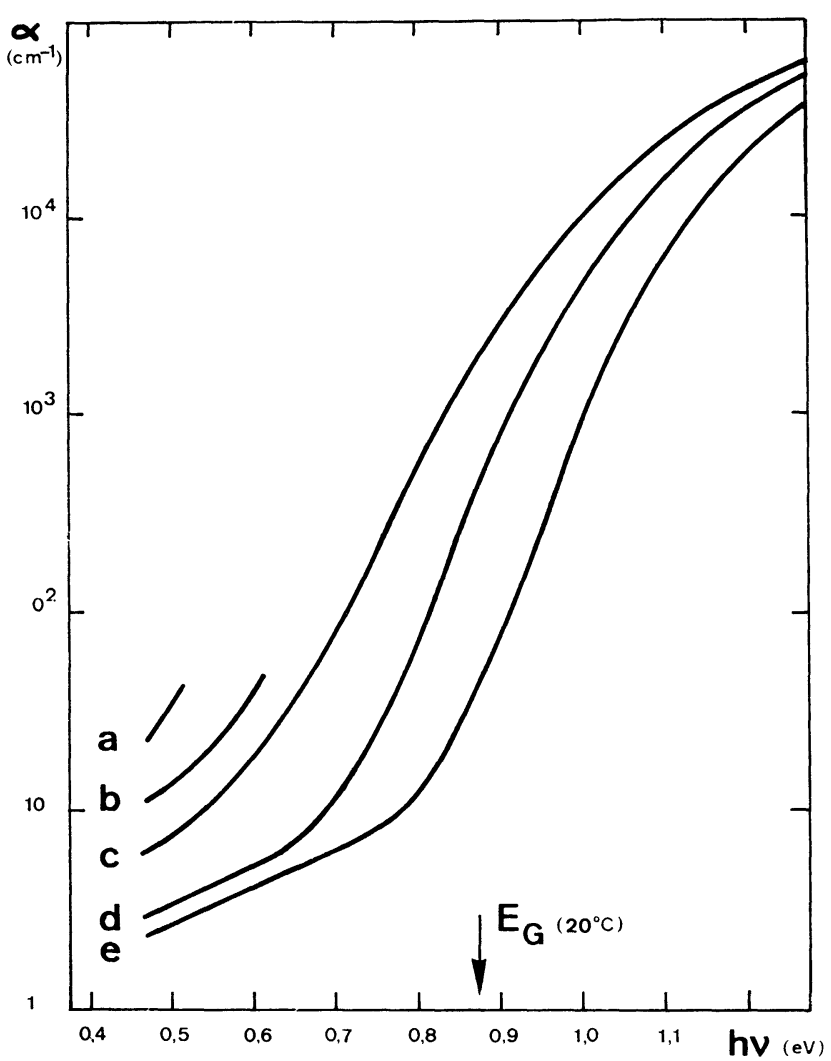

Fig. 3. - Coefficient d'absorption en fonction de l'énergie pour le composé vitreux $\mathrm{As}_{50} \mathrm{Te}_{50}$ à diverses températures; $a$ ) $450 \mathrm{~K}$, b) $425 \mathrm{~K}$, c) $400 \mathrm{~K}$, d) $300 \mathrm{~K}$, e) $130 \mathrm{~K}$. Des échantillons d'épaisseur variable entre $400 \AA$ et $1 \mathrm{~cm}$ ont été utilisés pour chacune de ces courbes (d'après [28]).

- pour $E>E_{\mathrm{G}}(T)$, on a

$$
\alpha \propto \frac{\left[h v-E_{G}(T)\right]^{2}}{h v}
$$

$E_{\mathrm{G}}(T)$ peut être obtenu par extrapolation à absorption nulle de $\sqrt{\alpha h v}=f(h v)$ et correspond généralement à une absorption de $10^{3} \mathrm{~cm}^{-1}$ à la température ordinaire. Ce sont ces valeurs de $E_{\mathrm{G}}$ qui figurent au tableau I.

- pour $E<E_{\mathrm{G}}$, on a d'abord une absorption à variation exponentielle puis dans le domaine

$$
10^{-2}<\alpha<10 \mathrm{~cm}^{-1}
$$

une absorption beaucoup plus faible, liée à la faible densité d'états localisés au milieu du gap. Dans ce domaine, si la température augmente, il y a absorption par les porteurs libres et $\alpha$ dépend de la température par une loi d'Arrhénius $\left(^{1}\right)$. Cependant, le terme préexponentiel est toujours plus faible que pour les composés cristallins correspondants car il y a très peu de porteurs libres; c'est pourquoi certains V.S. C.

(1) On suppose ici que le matériau ne présente pas d'impureté ou d'hétérogénéité, généralement à l'origine d'une absorption suffisamment forte aux grandes longueurs d'onde pour masquer complètement cette dépendance en température. suffisamment purs peuvent être utilisés en optique infrarouge de 3 à $14 \mu$ jusqu'à des températures assez élevées [1].

2.3 Photoconductivité. - La courbe de photoconductivité spectrale à une température donnée est voisine de la courbe d'absorption optique [5]. En fonction de la température, il y a un maximum à une température $T_{0}$ qui sépare des régimes de conduction différents [6] : à haute température, ce sont les porteurs dans les états étendus qui conduisent le courant ; la décroissance du photocourant en fonction de la température résulte de la diminution corrélative de la mobilité des porteurs. A basse température, au contraire, la conduction se fait essentiellement par hopping; l'augmentation du photocourant en fonction de la température reflète la croissance du nombre de porteurs. $T_{0}$ dépend au premier ordre du gap du matériau (Fig. 4) : pour les matériaux à grand gap (verres à base de soufre ou de sélénium par exemple), $T_{0}$ est supérieur à la température ordinaire ; pour les verres à base de tellure, au contraire, $T_{0}$ est voisin de $-50^{\circ} \mathrm{C}$ [7].

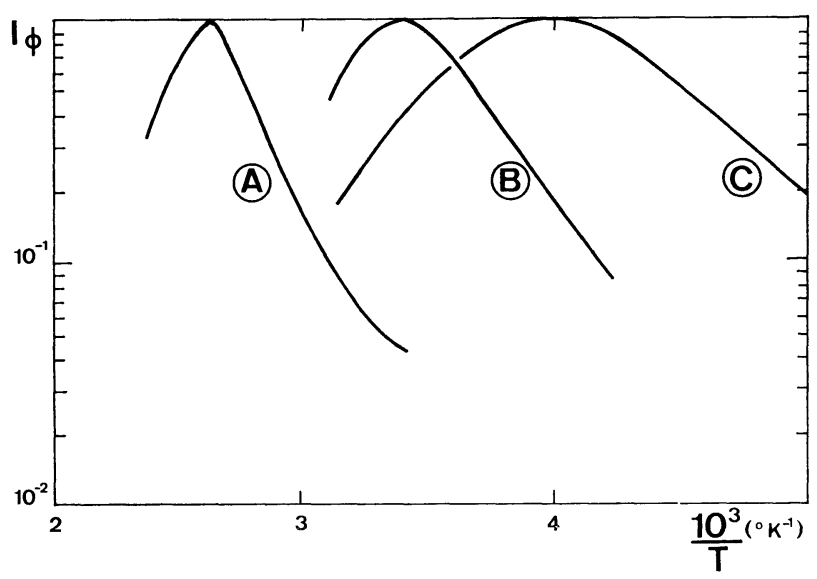

Fig. 4. - Variation du photocourant (unités arbitraires) en fonction de la température pour des V. S. C. à gap de photoconductivité très différents; (A): $\operatorname{As}_{2} \operatorname{Se}_{3}\left(E_{\varphi}=1,7 \mathrm{eV}\right)$ d'après [30] ; (B) $\mathrm{As}_{38} \mathrm{Te}_{38} \mathrm{Se}_{24}\left(E_{\varphi}=1,14 \mathrm{eV}\right)$ d'après [7] ; (C) $\mathrm{As}_{20} \mathrm{Te}_{80}\left(\mathrm{E}_{\varphi}=0,82 \mathrm{eV}\right)$ d'après [7].

3. Applications optiques. - Nous n'envisagerons ici que les chalcogénures ; ceux-ci peuvent en effet être préparés dans l'état idéal aussi bien sous forme massive que sous forme de couche mince, par opposition au silicium ou germanium amorphe dont les propriétés dépendent de l'histoire thermique.

3.1 Stockage OPTIQUe DE L'INFORMATION. - Si l'on apporte localement une quantité d'énergie judicieusement choisie, il est possible de modifier l'ordre local d'un V.S. C. Inversement, on peut décrire le chemin inverse, généralement par fusion, puis refroidissement et amorphisation. Ces transitions peuvent être obtenues par impulsion de courant [2] ou par faisceau de particules (électrons, neutrons, photons...). C'est en fait l'adressage optique qui est le plus commode et le plus facile à mettre en œuvre ; en outre, il n'implique 
pas la présence d'électrodes, susceptibles d'entraîner, par diffusion, un vieillissement prématuré des dispositifs.

Le contraste optique à la lecture dépend de la différence de gap entre le matériau amorphe de départ et le matériau irradié ; il faut donc distinguer trois classes de chalcogénures : les verres à base de tellure, ceux à base de sélénium et enfin ceux à base de soufre.

3.1.1 Verres à base de tellure. - De nombreux systèmes à base de tellure peuvent être déposés en couche mince sous forme vitreuse [8]. Le gap optique est voisin de $0,9 \mathrm{eV}$ et par chauffage au-dessus du point de ramollissement, il y a précipitation de tellure pur ( $g a p \simeq 0,4 \mathrm{eV})$. Ce chauffage peut être obtenu par impulsion lumineuse et il en résulte un contraste optique élevé, aussi bien en réflexion qu'en transmission entre la matrice amorphe et la région microcristallisée [9].

En fait, malgré la viscosité relativement faible du matériau, le temps d'incubation pour la nucléation du tellure est voisin de $1 \mu \mathrm{s}$, si bien que ce mode d'inscription appelé mode direct n'est pas adapté aux fréquences élevées. En outre le chauffage n'est pas adiabatique car, à cause de la longueur du pulse, il y a déperdition de chaleur latéralement et vers le substrat ; c'est pourquoi la sensibilité est aussi faible que $100 \mathrm{~nJ} / \mu^{2}[10]$.

En fait, ces matériaux sont utilisés plus avantageusement en mode inverse [11]. On prépare le matériau par évaporation sur un substrat à une température telle qu'il se forme une couche composite de microcristaux de tellure dans une matrice amorphe. Si on ajuste l'énergie des impulsions de telle façon qu'il y ait fusion locale, il y a amorphisation après le passage de l'impulsion. La résolution, la définition des spots et le contraste optique sont les mêmes que pour le mode direct, mais la fréquence d'inscription peut être aussi élevée que la puissance du laser le permet car la cinétique de nucléation du tellure n'intervient plus; il en résulte un échauffement quasi adiabatique, d'où une sensibilité bien meilleure que dans le mode direct, de l'ordre de $2 \mathrm{~nJ} / \mu^{2}$ pour une couche de $1000-2000 \AA$ d'épaisseur [8]. Le contraste optique dans la partie visible du spectre est voisin de 2 en transmission et de $40 \%$ en réflexion (Fig. 5). La capacité de la mémoire peut atteindre $10^{7} \mathrm{bits} / \mathrm{cm}^{2}$. En outre, il est possible d'effacer tout ou partie de l'information par nucléation du tellure dans les spots amorphisés [8].

3.1.2 Verres à base de sélénium. - Le processus d'inscription en mode inverse qui vient d'être décrit n'a pas été utilisé pour réaliser une mémoire optique avec les verres à base de sélénium car la différence de gap entre les phases amorphes et les phases cristallines est trop faible pour qu'on puisse obtenir des contrastes optiques suffisants. Cependant, en mode direct, certains verres à base de sélénium, contenant jusqu'à 40 at. \% d'arsenic présentent, à la différence des verres à base de tellure, une sensibilité acceptable. Le processus d'inscription est complexe et n'a pas reçu à ce

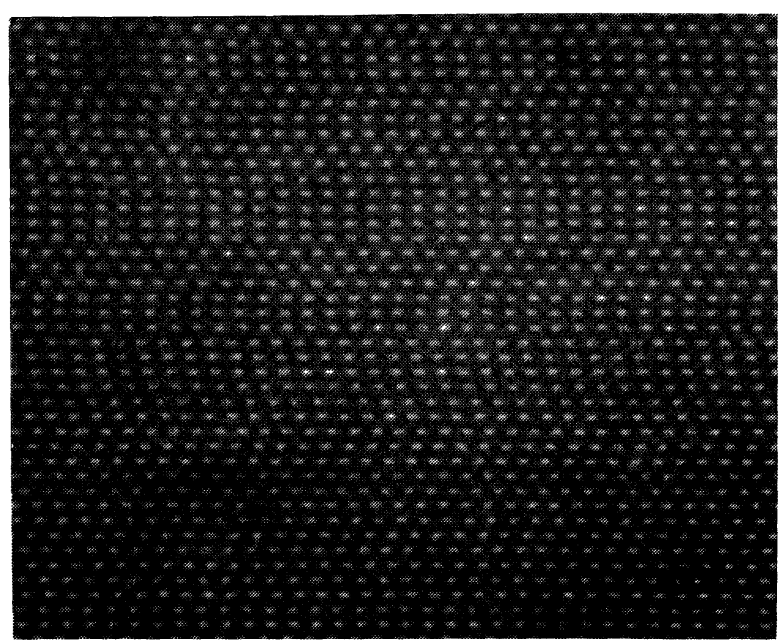

(a)

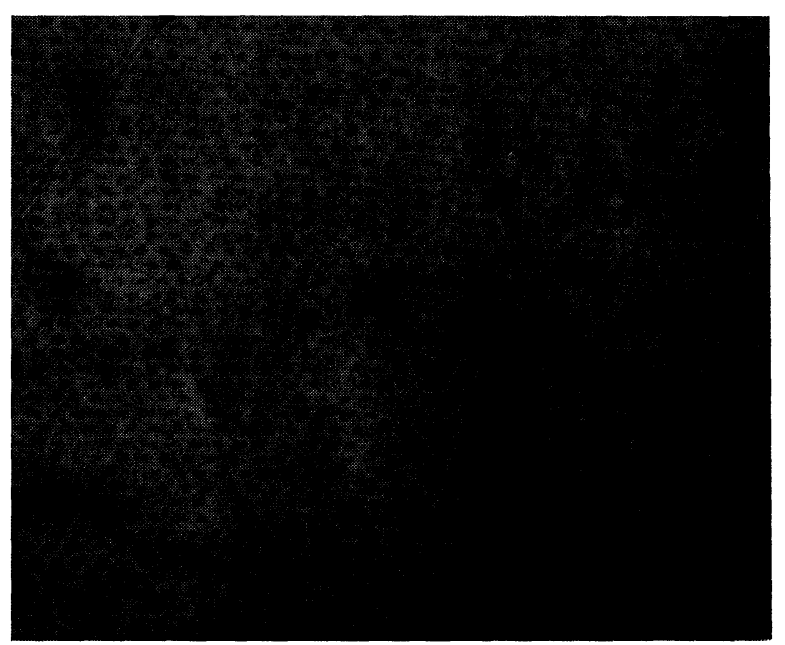

(b)

FIG. 5. - Micrographies d'une série de traces obtenues avec un laser argon ionisé sur une couche mince de composition $\mathrm{Ge}_{18} \mathrm{Te}_{82}$ et de $1000 \AA$ d'épaisseur ; $a$ ) en transmission (contraste $\simeq 2$ ), b) en réflexion (contraste $\simeq 40 \%$ ). La largeur des spots est $1 \mu$, la sensibilité $1 \mathrm{~nJ} /$ bit et la capacité $1,5 \times 10^{7}$ bits $\mathrm{cm}^{-2}$.

jour d'explication complètement satisfaisante: on observe, après irradiation avec une longueur d'onde $\leqslant 6500 \AA$, un déplacement du bord de bande d'absorption vers les plus grandes longueurs d'onde, par suite :

- d'une photodissociation des entités moléculaires -As-As- avec précipitation de l'arsenic ; la sensibilité et le contraste sont d'autant plus grands que la teneur en arsenic est plus élevée [12] ;

- d'une polymérisation des anneaux mixtes $\mathrm{Se}_{x} \mathrm{As}_{8-x}$ [13] qui existent dans le matériau même pour des teneurs en arsenic aussi élevées que $40 \%$. Cette polymérisation est initiée en partie thermiquement et en partie par photodissociation suivant le mécanisme mis en évidence par Dresner et Stringfellow [14].

Il a été montré, tout au moins dans le cas du sélénium pur, que le processus de polymérisation est réversible par cyclage autour de la température de polyméri- 
sation voisine de $50^{\circ} \mathrm{C}$ [15]. Un effacement complet de la mémoire nécessite cependant une fusion du matériau et une dissolution des précipités d'arsenic. Le contraste est au mieux égal à $40 \%$ en transmission [16] mais la résolution, la définition des spots et la sensibilité sont voisines de ce qu'on peut obtenir en mode inverse pour les verres à base de tellure. On peut augmenter le contraste par dissolution sélective des parties polymérisées si la mémoire a uniquement une utilisation mémoire-morte. Enfin, on peut envisager la réalisation d'hologrammes de phase ou de guide d'onde en mettant à profit la variation d'indice de réfraction lié au RedShift du bord de bande d'absorption.

3.1.3 Verres à base de soufre. - Comme pour les verres à base de sélénium, l'utilisation de ces matériaux pour une inscription en mode inverse n'est pas intéressante. De même, en mode direct, l'irradiation de ces matériaux avec une longueur d'onde $\leqslant 5500 \AA ̊$ conduit à un Red-shift du bord de bande d'absorption optique. Il ne peut s'agir ici de polymérisation car la température de polymérisation du soufre est voisine de $160^{\circ} \mathrm{C}$. Le phénomène a été interprété comme une photodissociation des entités moléculaires -As-S- avec précipitation de soufre [17]. Il en résulte des variations d'indice locales pouvant atteindre $2 \%$ ce qui a permis de réaliser des hologrammes présentant une efficacité de diffraction de $80 \%$ [18]. La résolution peut atteindre 10000 lignes $/ \mathrm{mm}$, mais la sensibilité n'est pas très élevée $\left(\simeq 75 \mathrm{~nJ} / \mu^{2}\right)$.

Cette mauvaise sensibilité peut néanmoins être considérablement améliorée dans l'utilisation mémoire morte si l'on dispose sur le matériau une couche d'argent de faible épaisseur $(\simeq 100 \AA)$ [19]. Dans ce cas il n'y a plus de précipitation de soufre, mais réaction du soufre avec l'argent et formation de $\mathrm{Ag}_{2} \mathrm{~S}$ transparent. Il en résulte non seulement une sensibilité accrue $\left(\simeq 0,7 \mathrm{~nJ} / \mu^{2}\right)$, mais aussi un excellent contraste optique. On peut ainsi réaliser des plaques photographiques, certes 1000 fois moins sensibles que les émulsions d'halogénures d'argent, mais aussi beaucoup moins chères (faible quantité d'argent, dépôt sous vide...). La principale utilisation semble être actuellement la réalisation de circuits imprimés (Fig. 6).

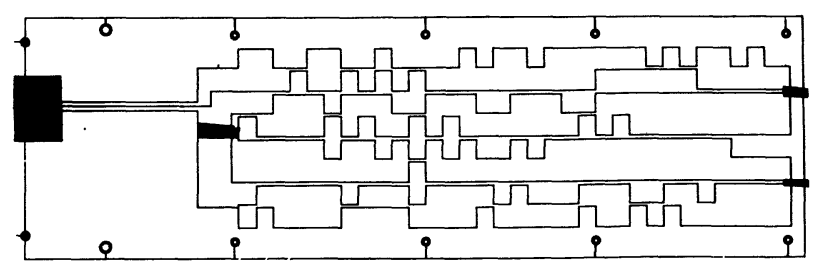

FIG. 6. - Circuit imprimé réalisé par exposition d'un sandwich $\mathrm{Ag} / \mathrm{As}_{2} \mathrm{~S}_{3}$. Les parties éclairées forment $\mathrm{Ag}_{2} \mathrm{~S}$ transparent. Du cuivre peut ensuite être déposé électrolytiquement sur l'argent qui n'a pas réagi de façon à former un circuit conducteur (communication personnelle de J. Malinovski).

3.2 APplications OPTIQUeS BASÉES SUR LA PHOTOCONDUCTIVITÉ. - 3.2.1 Xérographie. - Par absorption optique dans le sélénium et ses alliages, il y a formation de paires électron-trou qui peuvent être séparées par un champ électrique. L'efficacité quantique de photogénération, c'est-à-dire le nombre de paires créées par photon absorbé, est voisine de 1 au voisinage de la température ambiante pour $\lambda \leqslant 5600 \AA$ et un champ $\geqslant 10^{4} \mathrm{~V} . \mathrm{cm}^{-1}[20]$.

Ce sont ces conditions qui sont réalisées dans les machines Xérographiques. Le film photoconducteur déposé sur un substrat métallique au potentiel zéro est chargé positivement en surface par décharge corona (Fig. 7). Le document à reproduire est projeté sur le

(1)
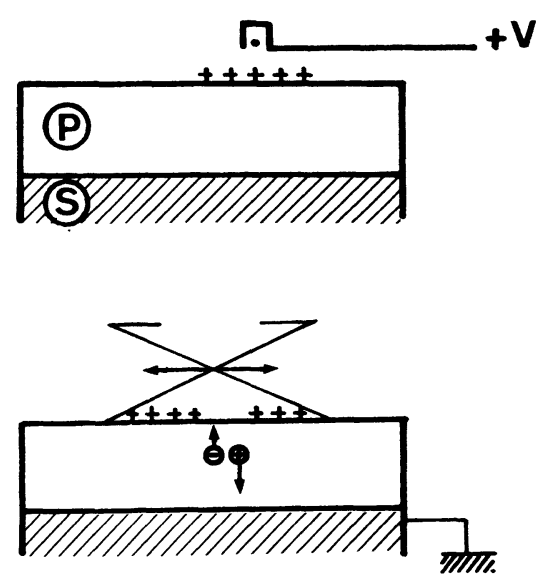

(3)

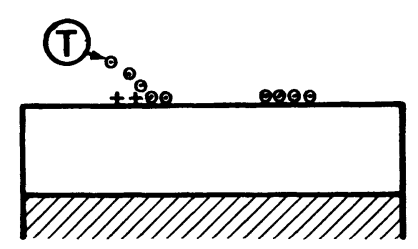

(4)

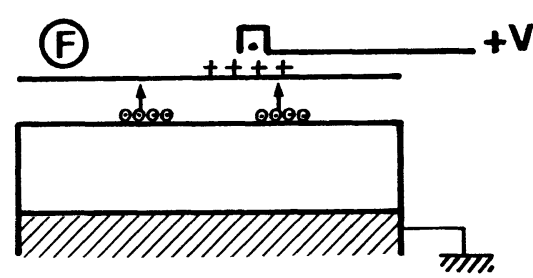

FIG. 7. - Principe du processus Xérographique (d'après [20]) : (1) Sensibilisation du photoconducteur $P$ déposé sur le substrat $S$. (2) Exposition. (3) Développement de l'image de charges par dépôt de Toner T. (4) Transfert de l'image sur une feuille de papier $F$.

film et des paires électron-trou sont créées au voisinage de la surface par les photons réfléchis sur les parties claires du document. Les trous migrent vers le substrat tandis que les électrons neutralisent les charges à la surface. Des particules de carbone dispersées dans une matière plastique chargée négativement sont alors projetées sur le film, adhérant ainsi aux zones non déchargées, puis sont transférées sur un papier chargé positivement. L'image est enfin fixée thermiquement. La résistivité du sélénium amorphe est égale à 
$10^{16} \Omega . \mathrm{cm}$ à $20^{\circ} \mathrm{C}$; elle est suffisante pour qu'il n'y ait pas de diffusion latérale des charges : la conduction se fait uniquement par hopping. De ce point de vue, les verres à base de soufre seraient préférables aux verres à base de sélénium, mais l'efficacité quantique de photogénération est beaucoup plus faible. Inversement, les verres au tellure, bien que plus panchromatiques que les verres au sélénium, ont une résistivité trop faible pour qu'on puisse les utiliser pour le stockage d'images de charges ; c'est également pour la même raison qu'on ne peut utiliser le sélénium cristallin.

3.2.2 Tubes de prise de vues. - C'est le même principe qui est utilisé dans certains tubes de prise de vues pour caméra de télévision. Dans ce cas, l'image de charges est lue séquentiellement par un faisceau électronique.

En fait, le sélénium pur a une faible stabilité thermique et une faible sensibilité dans le rouge. On peut augmenter considérablement sa stabilité par dopage à l'arsenic sans toutefois dépasser une teneur de $15 \%$ sinon la mobilité des électrons devient trop faible et il reste une image latente après interruption du faisceau lumineux. En outre, on peut augmenter la sensibilité dans le rouge en dopant avec du tellure au voisinage de l'électrode bloquante (Fig. 8), ce qui permet de conserver une résolution élevée. C'est sur ce principe qu'est réalisé le tube Saticon (Sélénium Arsenic Tellure SemI Conducteur) sensible dans le visible jusqu'à $0,7 \mu[21]$.

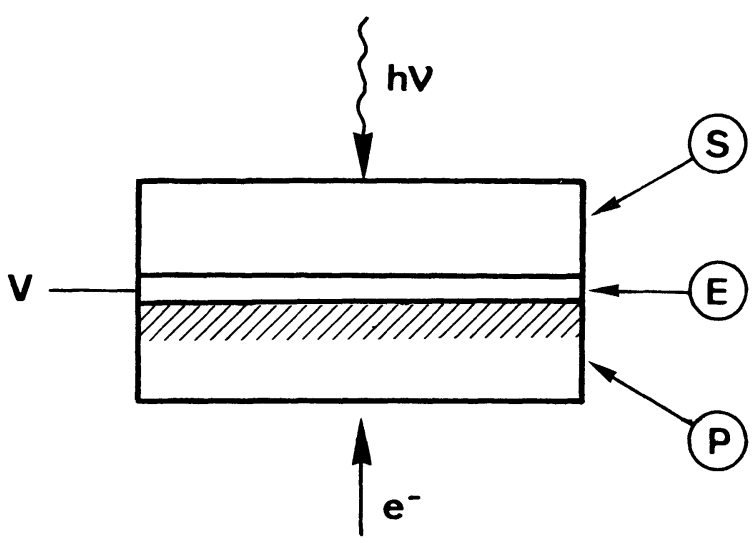

Fig. 8. - Représentation schématique de la structure du tube Saticon (d'après [21]). (S) désigne le substrat transparent sur lequel sont déposées l'électrode semi-transparente $(\mathrm{E})$ polarisée positivement et la couche photoconductrice $(\mathrm{P})$ constituée de sélénium stabilisé par l'arsenic. La partie grisée désigne la zone du photoconducteur dopée avec du tellure.

On peut aussi utiliser l'image latente dans la couche photo-sensible pour adresser une couche active optiquement et constituée soit de cristaux liquides, soit d'un corps cristallin présentant un effet électro-optique (effet Pockels par exemple). C'est cette association photoconducteur-cristal électro-optique qui est mise en œuvre dans le convertisseur optique Phototitus [22]. Grâce au caractère ambipolaire du photoconducteur, on peut additionner ou soustraire algébriquement des images

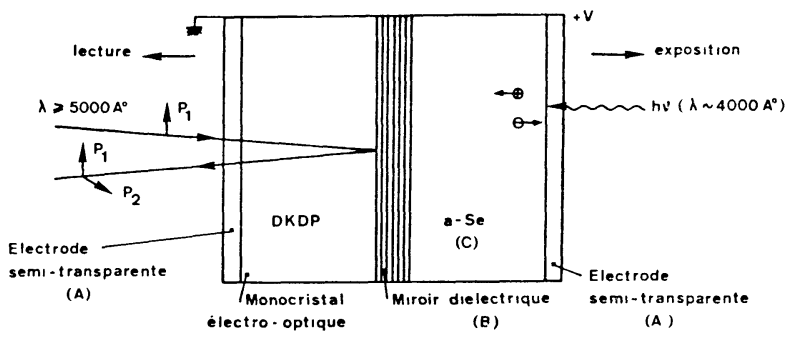

(a)
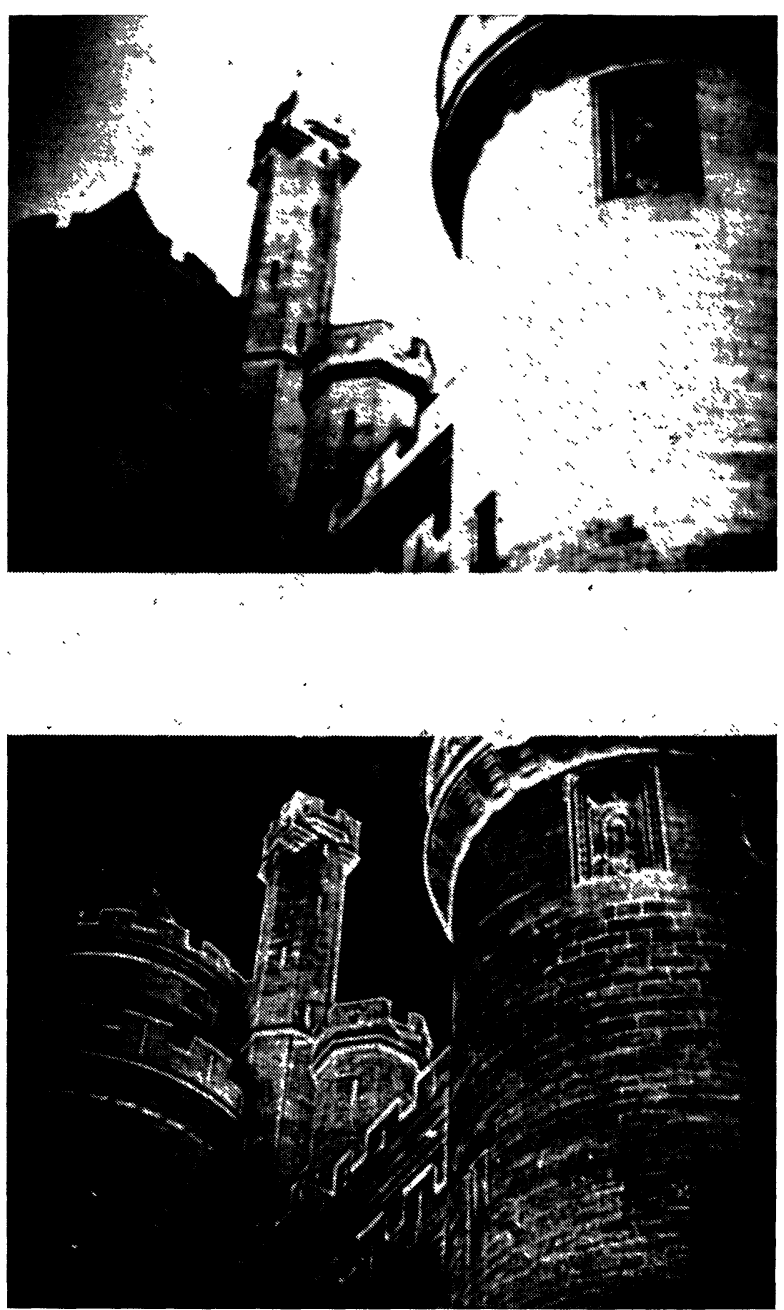

(b)

FIG. 9. - a) Coupe du tube Phototitus montrant le principe d'utilisation. La partie photosensible (C) est déposée sur un substrat de DKDP et est isolée optiquement de celui-ci par le miroir (B). La formation d'une image latente en (C) met sous tension le cristal électro-optique, ce qui entraîne une polarisation elliptique $P_{1}-P_{2}$ de la lumière de lecture polarisée rectilignement $\left(\mathrm{P}_{1}\right)$. b) Rehaussement des contours d'une image par différentiation du $1^{\text {er }}$ ordre omnidirectionnelle (d'après [23]).

(Fig. 9). On peut aussi faire de la conversion photonphoton permettant de modifier la longueur d'onde des rayons $X$ jusqu'à l'infrarouge proche [23]. Dans tous les cas, il est possible d'effectuer un traitement de l'image en lumière cohérente, par exemple du type filtrage de fréquences spatiales ou corrélation optique entre deux images. Les mêmes problèmes que pour le 
tube Saticon se posent en ce qui concerne la stabilité thermique et la sensibilité dans le rouge, mais ici les solutions sont moins immédiates car il est nécessaire qu'un dopage éventuel conserve aux électrons ainsi qu'aux trous une mobilité $\mu$ et une durée de vie $\tau$ telles que $\mu \tau \geqslant 10^{-7} \mathrm{~cm}^{2} . \mathrm{V}^{-1}$ de façon à ce que le parcours moyen de chacun des porteurs avant piégeage dans un piège profond soit au moins égal à l'épaisseur de la couche sensible : cette propriété est fondamentale pour la symétrie du tube, donc pour son utilisation en traitement d'images. Il semble qu'un dopage mixte à l'arsenic et à l'iode, ayant individuellement des effets opposés sur le produit $\mu . \tau$ pourrait conserver au sélénium les propriétés qui le caractérisent, tout en améliorant sa stabilité thermique et sa sensibilité dans le rouge.

3.2.3 Photodétecteurs rapides. - Certains composés du sélénium et du tellure présentent un maximum du photocourant à une température $T_{0}$ voisine de la température ordinaire. Si une couche mince de cette composition est placée entre deux contacts injectants, le gain de photoconduction est suffisamment élevé et le temps de réponse suffisamment court [7] pour permettre la réalisation d'ensembles comportant un très grand nombre de photodétecteurs dont on mesure séquentiellement le photocourant par un dispositif de multiplexage crossbar. Une propriété essentielle de ces dispositifs est de pouvoir être balayés électriquement à une vitesse quelconque entre $1 \mathrm{kbit} / \mathrm{s}$ et $1 \mathrm{Mbit} / \mathrm{s}$ sans modification du signal de photocourant recueilli car, grâce à la valeur élevée du gain de photoconduction, on peut mesurer la valeur instantanée du photocourant alors que les dispositifs classiques à photodiodes opèrent par intégration des charges. La résolution peut atteindre 6 points $/ \mathrm{mm}$ et on peut déposer le matériau sur des dimensions aussi grandes que $21 \mathrm{~cm}$, avec une uniformité meilleure que $5 \%$ [24]. En outre, le matériau est photosensible sans qu'il soit nécessaire de l'activer thermiquement ou chimiquement contrairement aux photoconducteurs cristallins de type CdS.

\subsection{Domaines nouveaux. - 3.3.1 Modulateurs et} déflecteurs acousto-optiques massifs. - Lorsqu'on est amené à choisir un matériau acousto-optique, non seulement le rendement de diffraction

$$
\frac{n^{6} \cdot p^{2}}{\rho \cdot v^{3}}
$$

intervient dans le choix, mais aussi l'absorption ultrasonore à fréquence élevée et l'absorption optique pour la longueur d'onde utilisée. Comme les coefficients acousto-optiques $p$ varient généralement très peu d'un corps à l'autre, quel que soit son état (oxyde, semiconducteur, cristal, verre...), on est amené à rechercher des matériaux d'indice $n$ élevé, de faible vitesse du son $v$ et d'absorptions optique et acoustique les plus faibles possibles [25].

L'ensemble de ces propriétés ne peut être obtenu simultanément et il est nécessaire d'adopter un compro-
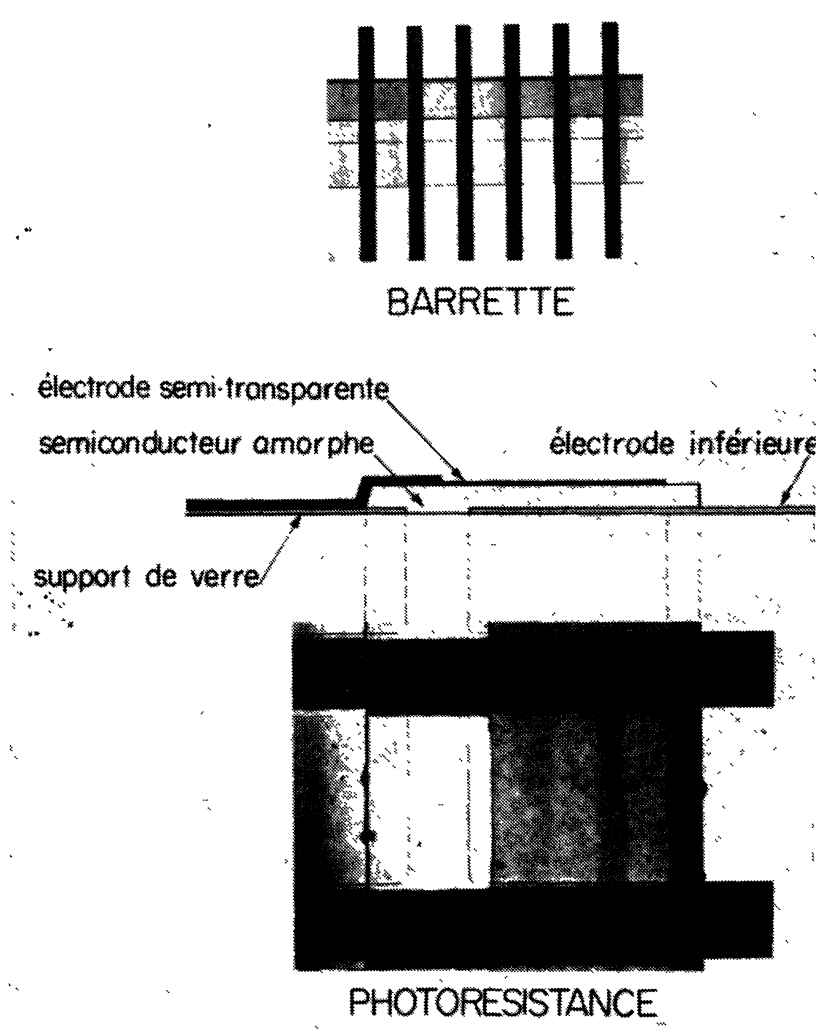

FIG. 10. - Portion d'un ensemble linéaire de photorésistances (barrette) au pas de $250 \mu$ et détail d'une photorésistance (d'après [24]).

mis. C'est ainsi que, dans le visible et le proche infrarouge, le niobate de lithium $\mathrm{LiNbO}_{3}$ et le molybdate de plomb $\mathrm{PbMoO}_{4}$ ont été retenus par suite essentiellement de leur très faible absorption acoustique et malgré une figure de mérite relativement faible. De même, dans l'infrarouge lointain, les meilleurs matériaux connus sont le germanium et surtout le tellure monocristallins, par suite essentiellement de leur indice de réfraction élevé. Or, dans tous les cas, les cristaux sont très difficiles soit à élaborer, soit à polir ou à conserver ; c'est ainsi que le tellure monocristallin, par suite de sa symétrie hexagonale et de l'existence de faibles liaisons Van der Waals perpendiculaires à l'axe $C$ est très difficile à préparer et à polir.

Or, il existe des matériaux isotropes très faciles à préparer et à conserver et qui présentent en outre des figures de mérite généralement élevées par suite d'un indice de réfraction important et d'une faible vitesse du son : ce sont les V. S. C. C'est ainsi que dans le visible (6 $328 \AA$ ) $\mathrm{As}_{2} \mathrm{~S}_{3}$ présente un bon rendement de diffraction [26] et une faible absorption optique ; cependant il peut y avoir une dissipation thermique trop élevée, qui entraîne une variation anisotrope de l'indice de réfraction et un élargissement du faisceau après déflexion ; en outre, il peut y avoir précipitation de soufre (cf. 2.1.1). Dans le proche infrarouge $(1,06 \mu)$, les verres du type $\mathrm{Ge}_{40} \mathrm{As}_{15} \mathrm{~S}_{45}$ présentent une figure de mérite élevée et une absorption ultra-sonore inférieure 
à $1 \mathrm{~dB} \cdot \mathrm{cm}^{-1}$ [27]. Enfin, dans l'infrarouge lointain, les verres à base de tellure peuvent être des matériaux de choix à cause de leur indice de réfraction élevé [28], de l'atténuation âcoustique faible, mais surtout de leur facilité de mise en œuvre avec de grandes dimensions pour certaines compositions [8]. En outre ils sont thermiquement et chimiquement stables, c'est-à-dire qu'ils peuvent - à la différence des verres au soufre ou au sélénium - être utilisés avec des lasers de puissance.

3.3.2 Optique intégrée. - Les mêmes matériaux que ceux qui ont été définis ci-dessus pour la modulation et la déflexion de la lumière, dans un domaine de longueurs d'onde adéquat, peuvent être déposés sous forme de films minces et utilisés pour la transmission, la modulation ou la déflexion de la lumière dans le même domaine de longueurs d'onde.

Ce domaine, très nouveau, a été très peu exploré ; la littérature ne cite que la réalisation d'un guide planaire $\mathrm{d}^{\prime} \mathrm{As}_{2} \mathrm{~S}_{3}$ amorphe fonctionnant à $1,064 \mu$ avec une absorption aussi faible que $0,4 \mathrm{~dB} / \mathrm{cm}$ pour le mode $\mathrm{TE}_{\mathrm{o}}$ [29]. Il est prévisible que la simplicité et le faible coût des méthodes de dépôt permettront aux V.S. C. d'amorcer de grands progrès dans ce domaine.

4. Conclusion. - Les idées et modèles établis pour les V.S.C. sont jusqu'à présent le résultat d'expériences. Peu de progrès théoriques ont été accomplis, essentiellement parce que le potentiel interatomique varie de façon aléatoire dans ces matériaux, si bien qu'il n'est pas possible d'utiliser la mécanique quantique pour effectuer des calculs rigoureux sur leur structure de bande. C'est pourquoi le modèle de Mott, généralement utilisé pour expliquer les propriétés des V.S. C. ne tient pas compte explicitement de l'ordre local ni des fluctuations du degré d'hybridation des orbitales dues au désordre.

Cependant, alors que, quasiment toutes les propriétés restent à expliquer, les V.S. C. ont déjà montré leur importance technologique. C'est sans nul doute le processus Xérographique qui a trouvé à ce jour la plus grande extension; mais le concept d'image latente qu'il utilise a une portée très générale et peut être utilisé dans des tubes de prise de vues ou de traitement d'images. En outre, les propriétés de changements de phase associés à l'existence d'un état vitreux métastable sont tout à fait spécifiques et permettent de réaliser soit des mémoires optiques recyclables de grande capacité, soit des mémoires holographiques, soit enfin des mémoires de type photographique. Enfin d'autres applications apparaissent lorsqu'on cherche à disposer d'une parfaite homogénéité ou reproductibilité des propriétés (photoconducteurs) ou d'une grande facilité de mise en œuvre et d'un faible coût (acousto-optique, optique intégrée...).

\section{Bibliographie}

[1] Hilton, A. R., Appl. Opt. 5 (1966) 1877.

[2] Neale, R. G., Nelson, D. C. et Moore, G. E., Electronics 43 (1970) 56.

[3] Theye, M. L., Proc. of the 5th Int. Conf. on Am. and Liq. Semiconductors, Garmisch-Partenkirchen (Taylor \& Francis London) 1975, p. 479.

[4] Stuke, J., J. Non-Cryst. Solids 4 (1970) 1.

[5] Pour une description détaillée voir par exemple Mотт, N. P. et Davis, E. A., Electronic Processes in Non-Crystalline Materials (Clarendon Press, Oxford) 1971.

[6] FAgen, E. A. et Fritzsche, A., J. Non-Cryst. Solids 4 (1970) 480.

[7] Cassanhiol, B., Cornet, J. et Rossier, D., Proc. of the 5th Int. Conf. on Am. and Liq. Semiconductors, Garmisch-Partenkirchen (Taylor \& Francis, London) 1974 p. 571.

[8] Cornet, J., Séminaires Chim. Etat Sol. 9,000 (ed. J. P. Suchet, Masson et Cie, Paris) 1975.

[9] Ovshinsky, S. R. et Fritzsche, J., Metall. Trans. 2 (1971) 641.

[10] Feinleib, J., de Neuville, J., Moss, S. C. et Ovshinsky, S. R., Appl. Phys. Lett. 18 (1971) 254.

[11] Von Gutfeld, R. J. et Chaudhari, P., J. Appl. Phys. 43 (1972) 4688.

[12] Gurevich, S. B., Ilyashenko, N. N., Kolomiets, B. T., Lyubin, V. M. et ShiLo, V. P., Phys. Stat. Sol. 26 (1974) K 127.

[13] Feinleib, J., Isawa, S., Moss, S. C., De Neufville, J. P. et Ovshinsky, S. R., J. Non-Cryst. Solids, 8-10 (1972) 909.

[14] Dresner, J. et Stringfellow, G. B., J. Phys. \& Chem. Solids 29 (1968) 303.
[15] Cornet, J. et Rossier, D., Proc. of the 5th Int. Conf. on Am. and Liq. Semiconductors, Garmisch-Partenkirchen (Taylor \& Francis London) 1974, p. 267.

[16] Asahara, Y. et Izumitani, T., J. Non-Cryst. Solids 16 (1974) 407.

[17] Pearson, A. D. et Bagley, B. G., Mater. Res. Bull. 6 (1971) 1041.

[18] Keneman, S. A., Appl. Phys. Lett. 19 (1971) 205.

[19] Malinovski, J., Thin Solid Films 13 (1972) 313.

[20] TabaK, M. D., Ing, S. W., Scharfe, M. E., IEEE Trans. Electron Devices 20 (1973) 132.

[21] Goto, N., Isozaki, Y., Shidara, K., Maruyama, E., HiRAI, T. et FuJITA, T., IEEE Trans. Electron Devices 21 (1974) 662.

[22] Grenot, M., Pergrale, J., Donjon, J. et Marie, G., Appl. Phys. Lett. 21 (1972) 83

[23] Donjon, J., Dumont, F., Grenot, M., Hazan, J. P., Marie, G. et Pergrale, J., IEEE Trans. Electron Devices 20 (1973) 1037.

[24] Cassanhiol, B., Cornet, J. et Rossier, D., $1^{\text {er }}$ Coll. Int. Pulvérisation Cathodique, Montpellier (1973).

[25] Voir par exemple Uchida, N. et NiIZeKI, N., Proc. IEEE 61 (1973) 1073.

[26] Dixon, R. W., J. Appl. Phys. 38 (1967) 5149.

[27] Krause, J. T., Kurkjian, C. R., Pinnow, D. A. et Sigety, E. A., Appl. Phys. Lett. 17 (1970) 367.

[28] Cornet, J. et Rossier, D., Phil. Mag. 27 (1973) 1335.

[29] Watts, R. K., De Wit, M. et Holton, W. C., Appl. Opt. 13 (1974) 2329.

[30] Kolomiets, R. T., Rukhlyader, Y. V. et Shilo, V. P., J. Non-Cryst. Solids 5 (1971) 389. 